\title{
Liter per Day per Microgram per Meter Squared
}

National Cancer Institute

\section{Source}

National Cancer Institute. Liter per Day per Microgram per Meter Squared. NCI

Thesaurus. Code C120736.

A unit of concentration equal to liter per day divided by microgram per meter squared. 